General Mathematics Vol. 28, No. 1 (2020), 67-83

DOI: $10.2478 / \mathrm{gm}-2020-0006$

sciendo

\title{
A refinement of Grüss inequality for the complex integral 1
}

Silvestru Sever Dragomir

\begin{abstract}
Assume that $f$ and $g$ are continuous on $\gamma, \gamma \subset \mathbb{C}$ is a piecewise smooth path parametrized by $z(t), t \in[a, b]$ from $z(a)=u$ to $z(b)=w$ with $w \neq u$ and the complex Čebyšev functional is defined by

$$
\mathcal{D}_{\gamma}(f, g):=\frac{1}{w-u} \int_{\gamma} f(z) g(z) d z-\frac{1}{w-u} \int_{\gamma} f(z) d z \frac{1}{w-u} \int_{\gamma} g(z) d z .
$$

In this paper we establish some Grüss type inequalities for $\mathcal{D}_{\gamma}(f, g)$ under some complex boundedness conditions for the functions $f$ and $g$.
\end{abstract}

2010 Mathematics Subject Classification: 26D15, 26D10, 30A10, 30A86.

Key words and phrases: Complex integral, Continuous functions, Holomorphic functions, Grüss inequality.

\section{Introduction}

For two Lebesgue integrable functions $f, g:[a, b] \rightarrow \mathbb{C}$, in order to compare the integral mean of the product with the product of the integral means, we consider the Čebyšev functional defined by

$$
C(f, g):=\frac{1}{b-a} \int_{a}^{b} f(t) g(t) d t-\frac{1}{b-a} \int_{a}^{b} f(t) d t \frac{1}{b-a} \int_{a}^{b} g(t) d t .
$$

\footnotetext{
${ }^{1}$ Received 13 February, 2020

Accepted for publication (in revised form) 22 April, 2020
} 
In 1934, G. Grüss [17] showed that

$$
|C(f, g)| \leq \frac{1}{4}(M-m)(N-n),
$$

provided $m, M, n, N$ are real numbers with the property that

$$
-\infty<m \leq f \leq M<\infty, \quad-\infty<n \leq g \leq N<\infty \quad \text { a.e. on }[a, b] .
$$

The constant $\frac{1}{4}$ is best possible in (1) in the sense that it cannot be replaced by a smaller one.

In [6], P. Cerone and S. S. Dragomir proved the following inequalities:

$$
|C(f, g)| \leq\left\{\begin{array}{r}
\inf _{\gamma \in \mathbb{R}}\|g-\gamma\|_{\infty} \frac{1}{b-a} \int_{a}^{b}\left|f(t)-\frac{1}{b-a} \int_{a}^{b} f(s) d s\right| d t \\
\inf _{\gamma \in \mathbb{R}}\|g-\gamma\|_{q} \frac{1}{b-a}\left(\int_{a}^{b}\left|f(t)-\frac{1}{b-a} \int_{a}^{b} f(s) d s\right|^{p} d t\right)^{\frac{1}{p}} \\
\text { where } p>1,1 / p+1 / q=1 .
\end{array}\right.
$$

For $\gamma=0$, we get from the first inequality in $(3)$

$$
|C(f, g)| \leq\|g\|_{\infty} \frac{1}{b-a} \int_{a}^{b}\left|f(t)-\frac{1}{b-a} \int_{a}^{b} f(s) d s\right| d t
$$

for which the constant 1 cannot be replaced by a smaller constant.

If $m \leq g \leq M$ for a.e. $x \in[a, b]$, then $\left\|g-\frac{m+M}{2}\right\|_{\infty} \leq \frac{1}{2}(M-m)$ and by the first inequality in (3) we can deduce the following result obtained by Cheng and Sun $[9]$

$$
|C(f, g)| \leq \frac{1}{2}(M-m) \frac{1}{b-a} \int_{a}^{b}\left|f(t)-\frac{1}{b-a} \int_{a}^{b} f(s) d s\right| d t .
$$

The constant $\frac{1}{2}$ is best in (5) as shown by Cerone and Dragomir in [7].

For other inequality of Grüss' type see [1]-[5], [7]-[16], [18]-[23] and [25]-[28].

In order to extend Grüss' inequality to complex integral we need the following preparations.

Suppose $\gamma$ is a smooth path parametrized by $z(t), t \in[a, b]$ and $f$ is a complex function which is continuous on $\gamma$. Put $z(a)=u$ and $z(b)=w$ with $u, w \in \mathbb{C}$. We define the integral of $f$ on $\gamma_{u, w}=\gamma$ as

$$
\int_{\gamma} f(z) d z=\int_{\gamma_{u, w}} f(z) d z:=\int_{a}^{b} f(z(t)) z^{\prime}(t) d t .
$$

We observe that that the actual choice of parametrization of $\gamma$ does not matter.

This definition immediately extends to paths that are piecewise smooth. Suppose $\gamma$ is parametrized by $z(t), t \in[a, b]$, which is differentiable on the intervals $[a, c]$ and $[c, b]$, then assuming that $f$ is continuous on $\gamma$ we define

$$
\int_{\gamma_{u, w}} f(z) d z:=\int_{\gamma_{u, v}} f(z) d z+\int_{\gamma_{v, w}} f(z) d z
$$


where $v:=z(c)$. This can be extended for a finite number of intervals.

We also define the integral with respect to arc-length

$$
\int_{\gamma_{u, w}} f(z)|d z|:=\int_{a}^{b} f(z(t))\left|z^{\prime}(t)\right| d t
$$

and the length of the curve $\gamma$ is then

$$
\ell(\gamma)=\int_{\gamma_{u, w}}|d z|=\int_{a}^{b}\left|z^{\prime}(t)\right| d t
$$

Let $f$ and $g$ be holomorphic in $G$, and open domain and suppose $\gamma \subset G$ is a piecewise smooth path from $z(a)=u$ to $z(b)=w$. Then we have the integration by parts formula

$$
\int_{\gamma_{u, w}} f(z) g^{\prime}(z) d z=f(w) g(w)-f(u) g(u)-\int_{\gamma_{u, w}} f^{\prime}(z) g(z) d z .
$$

We recall also the triangle inequality for the complex integral, namely

$$
\left|\int_{\gamma} f(z) d z\right| \leq \int_{\gamma}|f(z)||d z| \leq\|f\|_{\gamma, \infty} \ell(\gamma)
$$

where $\|f\|_{\gamma, \infty}:=\sup _{z \in \gamma}|f(z)|$.

We also define the $p$-norm with $p \geq 1$ by

$$
\|f\|_{\gamma, p}:=\left(\int_{\gamma}|f(z)|^{p}|d z|\right)^{1 / p} .
$$

For $p=1$ we have

$$
\|f\|_{\gamma, 1}:=\int_{\gamma}|f(z)||d z|
$$

If $p, q>1$ with $\frac{1}{p}+\frac{1}{q}=1$, then by Hölder's inequality we have

$$
\|f\|_{\gamma, 1} \leq[\ell(\gamma)]^{1 / q}\|f\|_{\gamma, p} .
$$

Suppose $\gamma \subset \mathbb{C}$ is a piecewise smooth path from $z(a)=u$ to $z(b)=w$ and $f: \gamma \rightarrow \mathbb{C}$ a continuous function on $\gamma$. Define the quantity:

$$
\begin{aligned}
\mathcal{P}_{\gamma}(f, \bar{f}) & =\frac{1}{\ell(\gamma)} \int_{\gamma}|f(z)|^{2}|d z|-\left|\frac{1}{\ell(\gamma)} \int_{\gamma} f(z)\right| d z||^{2} \\
& =\frac{1}{\ell(\gamma)} \int_{\gamma}\left|f(v)-\frac{1}{\ell(\gamma)} \int_{\gamma} f(z)\right| d z||^{2}|d v| \geq 0 .
\end{aligned}
$$

If $f$ and $g$ are continuous on $\gamma$, we consider the complex Čeby̌sev functional defined by

$$
\mathcal{D}_{\gamma}(f, g):=\frac{1}{w-u} \int_{\gamma} f(z) g(z) d z-\frac{1}{w-u} \int_{\gamma} f(z) d z \frac{1}{w-u} \int_{\gamma} g(z) d z .
$$

In this paper we establish some Grüss type inequalities for $\mathcal{D}_{\gamma}(f, g)$ under some complex boundedness conditions for the functions $f$ and $g$. 


\section{Some Preliminary Results}

We have the following equalities:

Lemma 1 Assume that the path $\gamma$ is piecewise smooth and $f: \gamma \rightarrow \mathbb{C}$ is continuous on $\gamma$. Then for $\phi, \Phi \in \mathbb{C}$ with $\phi \neq \Phi$ we have

(9) $\mathcal{P}_{\gamma}(f, \bar{f})=\operatorname{Re}\left[\left(\Phi-\frac{1}{\ell(\gamma)} \int_{\gamma} f(z)|d z|\right)\left(\frac{1}{\ell(\gamma)} \int_{\gamma} \overline{f(z)}|d z|-\bar{\phi}\right)\right]$

$$
-\frac{1}{\ell(\gamma)} \int_{\gamma} \operatorname{Re}[(\Phi-f(z))(\overline{f(z)}-\bar{\phi})]|d z|
$$

and, equivalently,

(10) $\mathcal{P}_{\gamma}(f, \bar{f})=\frac{1}{4}|\Phi-\phi|^{2}-\left|\frac{1}{\ell(\gamma)} \int_{\gamma} f(z)\right| d z\left|-\frac{\phi+\Phi}{2}\right|^{2}$

$$
-\frac{1}{\ell(\gamma)} \int_{\gamma} \operatorname{Re}[(\Phi-f(z))(\overline{f(z)}-\bar{\phi})]|d z| .
$$

Proof. We have

$$
\begin{array}{r}
I_{1}:=\operatorname{Re}\left[\left(\Phi-\frac{1}{\ell(\gamma)} \int_{\gamma} f(z)|d z|\right)\left(\frac{1}{\ell(\gamma)} \int_{\gamma} \overline{f(z)}|d z|-\bar{\phi}\right)\right] \\
=\operatorname{Re}\left[\frac{\Phi}{\ell(\gamma)} \int_{\gamma} \overline{f(z)}|d z|+\frac{\bar{\phi}}{\ell(\gamma)} \int_{\gamma} f(z)|d z|-\Phi \bar{\phi}-\left|\frac{1}{\ell(\gamma)} \int_{\gamma} f(z)\right| d z||^{2}\right] \\
=\operatorname{Re}\left(\frac{\Phi}{\ell(\gamma)} \int_{\gamma} \overline{f(z)}|d z|\right)+\operatorname{Re}\left(\frac{\bar{\phi}}{\ell(\gamma)} \int_{\gamma} f(z)|d z|\right) \\
-\operatorname{Re}(\Phi \bar{\phi})-\left|\frac{1}{\ell(\gamma)} \int_{\gamma} f(z)\right| d z||^{2}
\end{array}
$$

and

$$
\begin{aligned}
& I_{2}:=\frac{1}{\ell(\gamma)} \int_{\gamma} \operatorname{Re}[(\Phi-f(z))(\overline{f(z)}-\bar{\phi})]|d z| \\
&= \frac{1}{\ell(\gamma)} \int_{\gamma} \operatorname{Re}\left[\Phi \overline{f(z)}+\bar{\phi} f(z)-\Phi \bar{\phi}-|f(z)|^{2}\right]|d z| \\
&=\frac{1}{\ell(\gamma)} \int_{\gamma}\left[\operatorname{Re}(\Phi \overline{f(z)})+\operatorname{Re}(\bar{\phi} f(z))-\operatorname{Re}(\Phi \bar{\phi})-|f(z)|^{2}\right]|d z| \\
&=\operatorname{Re}\left(\frac{\Phi}{\ell(\gamma)} \int_{\gamma} \overline{f(z)}|d z|\right)+\operatorname{Re}\left(\frac{\bar{\phi}}{\ell(\gamma)} \int_{\gamma} f(z)|d z|\right) \\
&-\operatorname{Re}(\Phi \bar{\phi})-\frac{1}{\ell(\gamma)} \int_{\gamma}|f(z)|^{2}|d z| .
\end{aligned}
$$


Therefore

$$
I_{1}-I_{2}=\frac{1}{\ell(\gamma)} \int_{\gamma}|f(z)|^{2}|d z|-\left|\frac{1}{\ell(\gamma)} \int_{\gamma} f(z)\right| d z||^{2}
$$

proving the identity (9).

We have the equality for complex numbers

$$
\operatorname{Re}[(\Phi-w)(\bar{w}-\bar{\phi})]=\frac{1}{4}|\Phi-\phi|^{2}-\left|w-\frac{\phi+\Phi}{2}\right|^{2}
$$

for $w \in \mathbb{C}$, then by taking

$$
w=\frac{1}{\ell(\gamma)} \int_{\gamma} f(z)|d z|
$$

in this equality, we get

$$
\begin{aligned}
\operatorname{Re}\left[( \Phi - \frac { 1 } { \ell ( \gamma ) } \int _ { \gamma } f ( z ) | d z | ) \left(\frac{1}{\ell(\gamma)}\right.\right. & \left.\left.\int_{\gamma} \overline{f(z)}|d z|-\bar{\phi}\right)\right] \\
& =\frac{1}{4}|\Phi-\phi|^{2}-\left|\frac{1}{\ell(\gamma)} \int_{\gamma} f(z)\right| d z\left|-\frac{\phi+\Phi}{2}\right|^{2}
\end{aligned}
$$

and by (9) we obtain the desired result (10).

Suppose $\gamma \subset \mathbb{C}$ is a piecewise smooth path parametrized by $z(t), t \in \gamma$ from $z(a)=u$ to $z(b)=w$. Now, for $\phi, \Phi \in \mathbb{C}$, define the sets of complex-valued functions

$$
\bar{U}_{\gamma}(\phi, \Phi):=\{f: \gamma \rightarrow \mathbb{C} \mid \operatorname{Re}[(\Phi-f(z))(\overline{f(z)}-\bar{\phi})] \geq 0 \text { for each } z \in \gamma\}
$$

and

$$
\bar{\Delta}_{\gamma}(\phi, \Phi):=\left\{f: \gamma \rightarrow \mathbb{C}|| f(z)-\frac{\phi+\Phi}{2}\left|\leq \frac{1}{2}\right| \Phi-\phi \mid \text { for each } z \in \gamma\right\} .
$$

The following representation result may be stated.

Proposition 2 For any $\phi, \Phi \in \mathbb{C}, \phi \neq \Phi$, we have that $\bar{U}_{\gamma}(\phi, \Phi)$ and $\bar{\Delta}_{\gamma}(\phi, \Phi)$ are nonempty, convex and closed sets and

$$
\bar{U}_{\gamma}(\phi, \Phi)=\bar{\Delta}_{\gamma}(\phi, \Phi) .
$$

Proof. We observe that for any $w \in \mathbb{C}$ we have the equivalence

$$
\left|w-\frac{\phi+\Phi}{2}\right| \leq \frac{1}{2}|\Phi-\phi|
$$

if and only if

$$
\operatorname{Re}[(\Phi-w)(\bar{w}-\bar{\phi})] \geq 0
$$


This follows by the equality

$$
\frac{1}{4}|\Phi-\phi|^{2}-\left|w-\frac{\phi+\Phi}{2}\right|^{2}=\operatorname{Re}[(\Phi-w)(\bar{w}-\bar{\phi})]
$$

that holds for any $w \in \mathbb{C}$.

The equality (11) is thus a simple consequence of this fact.

On making use of the complex numbers field properties we can also state that:

Corollary 3 For any $\phi, \Phi \in \mathbb{C}, \phi \neq \Phi$, we have that

$$
\begin{aligned}
\bar{U}_{\gamma}(\phi, \Phi)= & \{f: \gamma \rightarrow \mathbb{C} \mid(\operatorname{Re} \Phi-\operatorname{Re} f(z))(\operatorname{Re} f(z)-\operatorname{Re} \phi) \\
& +(\operatorname{Im} \Phi-\operatorname{Im} f(z))(\operatorname{Im} f(z)-\operatorname{Im} \phi) \geq 0 \text { for each } z \in \gamma\} .
\end{aligned}
$$

Now, if we assume that $\operatorname{Re}(\Phi) \geq \operatorname{Re}(\phi)$ and $\operatorname{Im}(\Phi) \geq \operatorname{Im}(\phi)$, then we can define the following set of functions as well:

$$
\begin{aligned}
\bar{S}_{\gamma}(\phi, \Phi):=\{f: \gamma \rightarrow \mathbb{C} \mid & \operatorname{Re}(\Phi) \geq \operatorname{Re} f(z) \geq \operatorname{Re}(\phi) \\
& \text { and } \operatorname{Im}(\Phi) \geq \operatorname{Im} f(z) \geq \operatorname{Im}(\phi) \text { for each } z \in \gamma\}
\end{aligned}
$$

One can easily observe that $\bar{S}_{\gamma}(\phi, \Phi)$ is closed, convex and

$$
\emptyset \neq \bar{S}_{\gamma}(\phi, \Phi) \subseteq \bar{U}_{\gamma}(\phi, \Phi)
$$

Theorem 4 Assume that the path $\gamma$ is piecewise smooth and $f: \gamma \rightarrow \mathbb{C}$ is continuous on $\gamma$ and there exist $\phi, \Phi \in \mathbb{C}$ with $\phi \neq \Phi$ such that $f \in \bar{\Delta}_{\gamma}(\phi, \Phi)$. Then

$$
\mathcal{P}_{\gamma}(f, \bar{f}) \leq \operatorname{Re}\left[\left(\Phi-\frac{1}{\ell(\gamma)} \int_{\gamma} f(z)|d z|\right)\left(\frac{1}{\ell(\gamma)} \int_{\gamma} \overline{f(z)}|d z|-\bar{\phi}\right)\right] \leq \frac{1}{4}|\Phi-\phi|^{2}
$$

or, equivalently,

$$
\mathcal{P}_{\gamma}(f, \bar{f}) \leq \frac{1}{4}|\Phi-\phi|^{2}-\left|\frac{1}{\ell(\gamma)} \int_{\gamma} f(z)\right| d z\left|-\frac{\phi+\Phi}{2}\right|^{2} \leq \frac{1}{4}|\Phi-\phi|^{2}
$$

Proof. Since $f \in \bar{\Delta}_{\gamma}(\phi, \Phi)$, hence

$$
\operatorname{Re}[(\Phi-f(z))(\overline{f(z)}-\bar{\phi})] \geq 0 \text { for each } z \in \gamma \text {. }
$$

Therefore

$$
\frac{1}{\ell(\gamma)} \int_{\gamma} \operatorname{Re}[(\Phi-f(z))(\overline{f(z)}-\bar{\phi})]|d z| \geq 0
$$

and by Lemma 1 we deduce the desired result.

We have: 
Lemma 5 Suppose $\gamma \subset \mathbb{C}$ is a piecewise smooth path parametrized by $z(t), t \in \gamma$ from $z(a)=u$ to $z(b)=w$. If $f$ is continuous on $\gamma$, then for all $\lambda \in \mathbb{C}$ we have

$$
\mathcal{P}_{\gamma}(f, \bar{f})=\frac{1}{\ell(\gamma)} \int_{\gamma}\left(f(v)-\frac{1}{\ell(\gamma)} \int_{\gamma} f(z)|d z|\right)(\overline{f(v)}-\lambda)|d v| .
$$

Proof. We observe that

$$
\begin{aligned}
& \frac{1}{\ell(\gamma)} \int_{\gamma}\left(f(v)-\frac{1}{\ell(\gamma)} \int_{\gamma} f(z)|d z|\right)(\overline{f(v)}-\lambda)|d v| \\
& =\frac{1}{\ell(\gamma)} \int_{\gamma}|f(v)|^{2}|d v|-\frac{1}{\ell(\gamma)} \int_{\gamma} f(z)|d z| \frac{1}{\ell(\gamma)} \int_{\gamma} \overline{f(v)}|d v| \\
& -\lambda \frac{1}{\ell(\gamma)} \int_{\gamma}\left(f(v)-\frac{1}{\ell(\gamma)} \int_{\gamma} f(z)|d z|\right)|d v| \\
& =\frac{1}{\ell(\gamma)} \int_{\gamma}|f(v)|^{2}|d v|-\frac{1}{\ell(\gamma)} \int_{\gamma} f(z)|d z| \frac{1}{\ell(\gamma)} \overline{\left(\int_{\gamma} f(v)|d v|\right)} \\
& =\frac{1}{\ell(\gamma)} \int_{\gamma}|f(v)|^{2}|d v|-\left|\frac{1}{\ell(\gamma)} \int_{\gamma} f(z)\right| d z||^{2}
\end{aligned}
$$

for any $\lambda \in \mathbb{C}$, which proves (17).

We have:

Lemma 6 Suppose $\gamma \subset \mathbb{C}$ is a piecewise smooth path parametrized by $z(t), t \in \gamma$ from $z(a)=u$ to $z(b)=w$. If $f$ is continuous on $\gamma$ and there exists $c \in \mathbb{C}$ and $\rho>0$ such that

$$
f \in \bar{D}(c, \rho):=\{z \in \mathbb{C}|| z-c \mid \leq \rho\},
$$

then

$$
0 \leq \mathcal{P}_{\gamma}(f, \bar{f}) \leq \rho \frac{1}{\ell(\gamma)}\left|\int_{\gamma} f(v)-\frac{1}{\ell(\gamma)} \int_{\gamma} f(z)\right| d z|||d v|
$$

and

$$
\begin{aligned}
0 & \leq \mathcal{P}_{\gamma}^{2}(f, \bar{f}) \leq \rho^{2}\left(\frac{1}{\ell(\gamma)}\left|\int_{\gamma} f(v)-\frac{1}{\ell(\gamma)} \int_{\gamma} f(z)\right| d z|||d v|\right)^{2} \\
& \leq \rho^{2} \mathcal{P}_{\gamma}(f, \bar{f}) .
\end{aligned}
$$

Proof. For the equality (17) for $\lambda=\bar{c}$ we have

$$
\begin{aligned}
\mathcal{P}_{\gamma}(f, \bar{f}) & =\left|\frac{1}{\ell(\gamma)} \int_{\gamma}\left(f(v)-\frac{1}{\ell(\gamma)} \int_{\gamma} f(z)|d z|\right)(\overline{f(v)}-\bar{c})\right| d v|| \\
& \leq \frac{1}{\ell(\gamma)}\left|\int_{\gamma} f(v)-\frac{1}{\ell(\gamma)} \int_{\gamma} f(z)\right| d z|||\overline{f(v)}-\bar{c}||d v| \\
& =\frac{1}{\ell(\gamma)}\left|\int_{\gamma} f(v)-\frac{1}{\ell(\gamma)} \int_{\gamma} f(z)\right| d z|||f(v)-c||d v| \\
& \leq \rho \frac{1}{\ell(\gamma)}\left|\int_{\gamma} f(v)-\frac{1}{\ell(\gamma)} \int_{\gamma} f(z)\right| d z|||d v|,
\end{aligned}
$$


which proves (19).

Using Cauchy-Bunyakovsky-Schwarz integral inequality, we have

$$
\begin{aligned}
& \frac{1}{\ell(\gamma)}\left|\int_{\gamma} f(v)-\frac{1}{\ell(\gamma)} \int_{\gamma} f(z)\right| d z|||d v| \\
& \leq\left(\frac{1}{\ell(\gamma)} \int_{\gamma}\left|f(v)-\frac{1}{\ell(\gamma)} \int_{\gamma} f(z)\right| d z||^{2}|d v|\right)^{1 / 2} \\
& =\left(\frac{1}{\ell(\gamma)} \int_{\gamma}|f(z)|^{2}|d z|-\left|\frac{1}{\ell(\gamma)} \int_{\gamma} f(z)\right| d z||^{2}\right)^{1 / 2}
\end{aligned}
$$

where for the last equality we used (8).

From (19) and (21) we have

$$
0 \leq \mathcal{P}_{\gamma}(f, \bar{f}) \leq \rho \frac{1}{\ell(\gamma)}\left|\int_{\gamma} f(v)-\frac{1}{\ell(\gamma)} \int_{\gamma} f(z)\right| d z|||d v| \leq \rho\left[\mathcal{P}_{\gamma}(f, \bar{f})\right]^{1 / 2}
$$

which implies (20).

Corollary 7 Assume that the path $\gamma$ is piecewise smooth and $f: \gamma \rightarrow \mathbb{C}$ is continuous on $\gamma$ and there exist $\phi, \Phi \in \mathbb{C}$ with $\phi \neq \Phi$ such that $f \in \bar{\Delta}_{\gamma}(\phi, \Phi)$. Then

$$
0 \leq \mathcal{P}_{\gamma}(f, \bar{f}) \leq \frac{1}{2}|\Phi-\phi| \frac{1}{\ell(\gamma)}\left|\int_{\gamma} f(v)-\frac{1}{\ell(\gamma)} \int_{\gamma} f(z)\right| d z|||d v|
$$

and

$$
\begin{array}{r}
0 \leq \mathcal{P}_{\gamma}^{2}(f, \bar{f}) \leq \frac{1}{4}|\Phi-\phi|^{2}\left(\frac{1}{\ell(\gamma)} \int_{\gamma}\left|f(v)-\frac{1}{\ell(\gamma)} \int_{\gamma} f(z)\right| d z|||d v|\right)^{2} \\
\leq \frac{1}{4}|\Phi-\phi|^{2} \operatorname{Re}\left[\left(\Phi-\frac{1}{\ell(\gamma)} \int_{\gamma} f(z)|d z|\right)\left(\frac{1}{\ell(\gamma)} \int_{\gamma} \overline{f(z)}|d z|-\bar{\phi}\right)\right] \\
=\frac{1}{4}|\Phi-\phi|^{2}\left[\frac{1}{4}|\Phi-\phi|^{2}-\left|\frac{1}{\ell(\gamma)} \int_{\gamma} f(z)\right| d z\left|-\frac{\phi+\Phi}{2}\right|^{2}\right] \\
\leq \frac{1}{16}|\Phi-\phi|^{4} .
\end{array}
$$

\section{Refinements of Grüss' Inequality}

We start with the following identity of interest: 
Lemma 8 Suppose $\gamma \subset \mathbb{C}$ is a piecewise smooth path parametrized by $z(t), t \in \gamma$ from $z(a)=u$ to $z(b)=w$ with $w \neq u$. If $f$ and $g$ are continuous on $\gamma$, then

$$
\begin{aligned}
\mathcal{D}_{\gamma}(f, g) & =\frac{1}{2(w-u)^{2}} \int_{\gamma}\left(\int_{\gamma}(f(z)-f(w))(g(z)-g(w)) d w\right) d z \\
= & \frac{1}{2(w-u)^{2}} \int_{\gamma}\left(\int_{\gamma}(f(z)-f(w))(g(z)-g(w)) d z\right) d w \\
& =\frac{1}{2(w-u)^{2}} \int_{\gamma} \int_{\gamma}(f(z)-f(w))(g(z)-g(w)) d z d w .
\end{aligned}
$$

Proof. For any $z \in \gamma$ the integral $\int_{\gamma}(f(z)-f(w))(g(z)-g(w)) d w$ exists and

$$
\begin{gathered}
I(z):=\int_{\gamma}(f(z)-f(w))(g(z)-g(w)) d w \\
=\int_{\gamma}(f(z) g(z)+f(w) g(w)-g(z) f(w)-f(z) g(w)) d w \\
=f(z) g(z) \int_{\gamma} d w+\int_{\gamma} f(w) g(w) d w-g(z) \int_{\gamma} f(w) d w-f(z) \int_{\gamma} g(w) d w \\
=(w-u) f(z) g(z)+\int_{\gamma} f(w) g(w) d w-g(z) \int_{\gamma} f(w) d w-f(z) \int_{\gamma} g(w) d w .
\end{gathered}
$$

The function $I(z)$ is also continuous on $\gamma$, then the integral $\int_{\gamma} I(z) d z$ exists and

$$
\begin{aligned}
\int_{\gamma} I(z) d z=\int_{\gamma}\left[(w-u) f(z) g(z)+\int_{\gamma} f(w) g(w) d w\right. \\
\left.\quad-g(z) \int_{\gamma} f(w) d w-f(z) \int_{\gamma} g(w) d w\right] d z \\
=(w-u) \int_{\gamma} f(z) g(z) d z+(w-u) \int_{\gamma} f(w) g(w) d w \\
-\int_{\gamma} f(w) d w \int_{\gamma} g(z) d z-\int_{\gamma} g(w) d w \int_{\gamma} f(z) d z \\
=2(w-u) \int_{\gamma} f(z) g(z) d z-2 \int_{\gamma} f(z) d z \int_{\gamma} g(z) d z=2(w-u)^{2} \mathcal{P}_{\gamma}(f, g),
\end{aligned}
$$

which proves the first equality in (24).

The rest follows in a similar manner and we omit the details.

We have:

Lemma 9 Suppose $\gamma \subset \mathbb{C}$ is a piecewise smooth path parametrized by $z(t), t \in \gamma$ from $z(a)=u$ to $z(b)=w$ with $w \neq u$. If $f$ and $g$ are continuous on $\gamma$, then

$$
\left|\mathcal{D}_{\gamma}(f, g)\right| \leq \epsilon^{2}(\gamma)\left[\mathcal{P}_{\gamma}(f, \bar{f})\right]^{1 / 2}\left[\mathcal{P}_{\gamma}(g, \bar{g})\right]^{1 / 2},
$$

where $\epsilon(\gamma):=\frac{\ell(\gamma)}{|w-u|}$ can be interpreted as the deviation of the path $\gamma$ from the segment joining the points $u$ and $w$ in $\mathbb{C}$. 
Proof. Taking the modulus in the first equality in (24), we get

$$
\begin{aligned}
\left|\mathcal{D}_{\gamma}(f, g)\right|=\frac{1}{2|w-u|^{2}}\left|\int_{\gamma}\left(\int_{\gamma}(f(z)-f(w))(g(z)-g(w)) d w\right) d z\right| \\
\leq \frac{1}{2|w-u|^{2}} \int_{\gamma}\left|\int_{\gamma}(f(z)-f(w))(g(z)-g(w)) d w\right||d z|=: A
\end{aligned}
$$

Using the Cauchy-Bunyakovsky-Schwarz integral inequality, we have

$$
\begin{aligned}
\mid \int_{\gamma}(f(z)-f(w)) & (g(z)-g(w)) d w \mid \\
\leq & \left(\int_{\gamma}|f(z)-f(w)|^{2}|d w|\right)^{1 / 2}\left(\int_{\gamma}|g(z)-g(w)|^{2}|d w|\right)^{1 / 2}
\end{aligned}
$$

which implies that

$A$

$$
\begin{array}{r}
\leq \frac{1}{2|w-u|^{2}} \int_{\gamma}\left(\int_{\gamma}|f(z)-f(w)|^{2}|d w|\right)^{1 / 2}\left(\int_{\gamma}|g(z)-g(w)|^{2}|d w|\right)^{1 / 2}|d z| \\
=: B .
\end{array}
$$

By the Cauchy-Bunyakovsky-Schwarz integral inequality, we also have

$$
\begin{gathered}
\int_{\gamma}\left(\int_{\gamma}|f(z)-f(w)|^{2}|d w|\right)^{1 / 2}\left(\int_{\gamma}|g(z)-g(w)|^{2}|d w|\right)^{1 / 2}|d z| \\
\leq\left(\int_{\gamma}\left[\left(\int_{\gamma}|f(z)-f(w)|^{2}|d w|\right)^{1 / 2}\right]^{2}|d z|\right)^{1 / 2} \\
\times\left(\int_{\gamma}\left[\left(\int_{\gamma}|g(z)-g(w)|^{2}|d w|\right)^{1 / 2}\right]^{2}|d z|\right)^{1 / 2} \\
=\left(\int_{\gamma}\left(\int_{\gamma}|f(z)-f(w)|^{2}|d w|\right)|d z|\right)^{1 / 2}\left(\int_{\gamma}\left(\int_{\gamma}|g(z)-g(w)|^{2}|d w|\right)|d z|\right)^{1 / 2},
\end{gathered}
$$

which implies that

(26) $B \leq \frac{1}{2|w-u|^{2}}\left(\int_{\gamma}\left(\int_{\gamma}|f(z)-f(w)|^{2}|d w|\right)|d z|\right)^{1 / 2}$

$$
\times\left(\int_{\gamma}\left(\int_{\gamma}|g(z)-g(w)|^{2}|d w|\right)|d z|\right)^{1 / 2} .
$$


Now, observe that

$$
\begin{gathered}
\int_{\gamma}\left(\int_{\gamma}|f(z)-f(w)|^{2}|d w|\right)|d z| \\
=\int_{\gamma}\left(\int_{\gamma}\left(|f(z)|^{2}-2 \operatorname{Re}(f(z) \overline{f(w)})+|f(w)|^{2}\right)|d w|\right)|d z| \\
=\int_{\gamma}\left(\ell(\gamma)|f(z)|^{2}-2 \operatorname{Re}\left(f(z) \int_{\gamma} \overline{f(w)}|d w|\right)+\int_{\gamma}|f(w)|^{2}|d w|\right)|d z| \\
=\ell(\gamma) \int_{\gamma}|f(z)|^{2}|d z|-2 \operatorname{Re}\left(\int_{\gamma} f(z)|d z| \int_{\gamma} \overline{f(w)}|d w|\right)+\ell(\gamma) \int_{\gamma}|f(w)|^{2}|d w| \\
=2 \ell(\gamma) \int_{\gamma}|f(z)|^{2}|d z|-2 \operatorname{Re}\left(\int_{\gamma} f(z)|d z|\left(\int_{\gamma} f(w)|d w|\right)\right) \\
=2\left[\ell(\gamma) \int_{\gamma}|f(z)|^{2}|d z|-\left|\int_{\gamma} f(z)\right| d z||^{2}\right]=2 \ell^{2}(\gamma) \mathcal{P}_{\gamma}(f, \bar{f})
\end{gathered}
$$

and, similarly

$$
\int_{\gamma}\left(\int_{\gamma}|g(z)-g(w)|^{2}|d w|\right)|d z|=2 \ell^{2}(\gamma) \mathcal{P}_{\gamma}(g, \bar{g}) .
$$

Making use of (27) and (28), we get

$$
\begin{aligned}
B \leq \frac{1}{2|w-u|^{2}}\left[2 \ell^{2}(\gamma) \mathcal{P}_{\gamma}(f, \bar{f})\right]^{1 / 2}\left[2 \ell^{2}(\gamma) \mathcal{P}_{\gamma}(g, \bar{g})\right]^{1 / 2} & \\
& =\frac{\ell^{2}(\gamma)}{|w-u|^{2}}\left[\mathcal{P}_{\gamma}(f, \bar{f})\right]^{1 / 2}\left[\mathcal{P}_{\gamma}(g, \bar{g})\right]^{1 / 2},
\end{aligned}
$$

which proves the desired result (25).

Remark 1 For $g=f$ we have

$$
\mathcal{D}_{\gamma}(f, f)=\frac{1}{w-u} \int_{\gamma} f^{2}(z) d z-\left(\frac{1}{w-u} \int_{\gamma} f(z) d z\right)^{2}
$$

and by (25) we get

$$
\left|\mathcal{D}_{\gamma}(f, f)\right| \leq \epsilon^{2}(\gamma) \mathcal{P}_{\gamma}(f, \bar{f})
$$

For $g=\bar{f}$ we have

$$
\mathcal{D}_{\gamma}(f, \bar{f})=\frac{1}{w-u} \int_{\gamma}|f(z)|^{2} d z-\frac{1}{w-u} \int_{\gamma} f(z) d z \frac{1}{w-u} \int_{\gamma} \overline{f(z)} d z
$$

and by (25) we get

$$
\left|\mathcal{D}_{\gamma}(f, \bar{f})\right| \leq \epsilon^{2}(\gamma) \mathcal{P}_{\gamma}(f, \bar{f})
$$


Theorem 10 Suppose $\gamma \subset \mathbb{C}$ is a piecewise smooth path parametrized by $z(t), t \in \gamma$ from $z(a)=u$ to $z(b)=w$ with $w \neq u$. If $f$ and $g$ are continuous on $\gamma$ and there exists $\phi, \Phi, \psi, \Psi \in \mathbb{C}, \phi \neq \Phi, \psi \neq \Psi$ such that $f \in \bar{\Delta}_{\gamma}(\phi, \Phi)$ and $g \in \bar{\Delta}_{\gamma}(\psi, \Psi)$ then

$$
\begin{aligned}
\left|\mathcal{D}_{\gamma}(f, g)\right| & \leq \epsilon^{2}(\gamma)\left(\frac{1}{4}|\Phi-\phi|^{2}-\left|\frac{1}{\ell(\gamma)} \int_{\gamma} f(z)\right| d z\left|-\frac{\phi+\Phi}{2}\right|^{2}\right)^{1 / 2} \\
& \times\left(\frac{1}{4}|\Psi-\psi|^{2}-\left|\frac{1}{\ell(\gamma)} \int_{\gamma} g(z)\right| d z\left|-\frac{\psi+\Psi}{2}\right|^{2}\right)^{1 / 2} \\
& \leq \epsilon^{2}(\gamma)\left[\frac{1}{4}|\Phi-\phi||\Psi-\psi|\right. \\
\left.-\left|\frac{1}{\ell(\gamma)} \int_{\gamma} f(z)\right| d z\left|-\frac{\phi+\Phi}{2}\right|\left|\frac{1}{\ell(\gamma)} \int_{\gamma} g(z)\right| d z\left|-\frac{\psi+\Psi}{2}\right|\right] & \leq \frac{1}{4} \epsilon^{2}(\gamma)|\Phi-\phi||\Psi-\psi|
\end{aligned}
$$

Proof. The first inequality in (33) follows by Corollary 7 and Lemma 9 .

Using the elementary inequality

$$
\left(m^{2}-n^{2}\right)^{1 / 2}\left(p^{2}-q^{2}\right)^{1 / 2} \leq m p-n q
$$

that holds for $m \geq n \geq 0$ and $p \geq q \geq 0$, for the choices

$$
m=\frac{1}{2}|\Phi-\phi|, n=\left|\frac{1}{\ell(\gamma)} \int_{\gamma} f(z)\right| d z\left|-\frac{\phi+\Phi}{2}\right|
$$

and

$$
p=\frac{1}{2}|\Psi-\psi|, q=\left|\frac{1}{\ell(\gamma)} \int_{\gamma} g(z)\right| d z\left|-\frac{\psi+\Psi}{2}\right|
$$

we get the second inequality in (33).

The last part is obvious.

Remark 2 If there is information on the boundedness of only one function, namely $f \in \bar{\Delta}_{\gamma}(\phi, \Phi)$, where $g: \gamma \rightarrow \mathbb{C}$ is continuous, then we have the "premature" Grüss inequality

$$
\begin{aligned}
&\left|\mathcal{D}_{\gamma}(f, g)\right| \leq \epsilon^{2}(\gamma)\left(\frac{1}{4}|\Phi-\phi|^{2}-\left|\frac{1}{\ell(\gamma)} \int_{\gamma} f(z)\right| d z\left|-\frac{\phi+\Phi}{2}\right|^{2}\right)^{1 / 2} \\
& \times\left[\mathcal{P}_{\gamma}(g, \bar{g})\right]^{1 / 2}
\end{aligned}
$$




\section{Applications for Trapezoid Inequality}

Suppose $\gamma$ is a smooth path parametrized by $z(t), t \in[a, b]$ and $f$ holomorphic in $G$, an open domain and suppose $\gamma \subset G$. Put $z(a)=u$ and $z(b)=w$ with $u, w \in \mathbb{C}$. Using the integration by parts formula (6) twice we have

$$
\int_{\gamma_{u, v}}(z-v) f^{\prime}(z) d z=(v-u) f(u)-\int_{\gamma_{u, v}} f(z) d z
$$

and

$$
\int_{\gamma_{v, w}}(z-v) f^{\prime}(z) d z=(w-v) f(w)-\int_{\gamma_{v, w}} f(z) d z
$$

for any $v \in \gamma$.

If we add these two equalities, we get the generalized trapezoid equality

$$
\begin{aligned}
(v-u) & f(u)+(w-v) f(w)-\int_{\gamma} f(z) d z \\
= & \int_{\gamma_{u, v}}(z-v) f^{\prime}(z) d z+\int_{\gamma_{v, w}}(z-v) f^{\prime}(z) d z=\int_{\gamma}(z-v) f^{\prime}(z) d z
\end{aligned}
$$

with the above assumptions for $u, v$ and $w$ on $\gamma$.

If we take $v=\frac{u+w}{2}$, then we get the trapezoid equality

$$
(w-u) \frac{f(u)+f(w)}{2}-\int_{\gamma} f(z) d z=\int_{\gamma}\left(z-\frac{u+w}{2}\right) f^{\prime}(z) d z,
$$

which also can be written as

$$
\frac{f(u)+f(w)}{2}-\frac{1}{w-u} \int_{\gamma} f(z) d z=\mathcal{D}_{\gamma}\left(f^{\prime}, h\right)
$$

where $h(z)=z-\frac{u+w}{2}, z \in \mathbb{C}$, since $\int_{\gamma}\left(z-\frac{u+w}{2}\right) d z=0$.

If $f^{\prime} \in \bar{\Delta}_{\gamma}(\theta, \Theta)$ for some $\theta, \Theta \in \mathbb{C}$ with $\theta \neq \Theta$, then by (34) we get the following trapezoid inequality of interest:

$$
\begin{aligned}
\left|\frac{f(u)+f(w)}{2}-\frac{1}{w-u} \int_{\gamma} f(z) d z\right|=\mathcal{D}_{\gamma}\left(f^{\prime}, h\right) & \\
\leq \epsilon^{2}(\gamma)\left(\frac{1}{4}|\Theta-\theta|^{2}-\left|\frac{1}{\ell(\gamma)} \int_{\gamma} f^{\prime}(z)\right| d z \mid\right. & \left.-\left.\frac{\theta+\Theta}{2}\right|^{2}\right)^{1 / 2}\left[\mathcal{P}_{\gamma}(h, \bar{h})\right]^{1 / 2} \\
& \leq \frac{1}{2}|\Theta-\theta| \epsilon^{2}(\gamma)\left[\mathcal{P}_{\gamma}(h, \bar{h})\right]^{1 / 2},
\end{aligned}
$$

where

$$
\mathcal{P}_{\gamma}(h, \bar{h})=\frac{1}{\ell(\gamma)} \int_{\gamma}\left|z-\frac{u+w}{2}\right|^{2}|d z|-\left|\frac{1}{\ell(\gamma)} \int_{\gamma}\left(z-\frac{u+w}{2}\right)\right| d z||^{2} .
$$


If the path $\gamma$ is a segment $[u, w] \subset G$ connecting two distinct points $u$ and $w$ in $G$ then we write $\int_{\gamma} f(z) d z$ as $\int_{u}^{w} f(z) d z$. We then have

$$
\begin{gathered}
\epsilon(\gamma)=\frac{\ell(\gamma)}{|w-u|}=\frac{|w-u|}{|w-u|}=1, \\
\frac{1}{\ell([u, w])} \int_{u}^{w} f^{\prime}(z)|d z|=\frac{|w-u|}{|w-u|} \int_{0}^{1} f^{\prime}((1-t) u+t w) d t=\frac{f(w)-f(u)}{w-u}
\end{gathered}
$$

and

$$
\begin{gathered}
\mathcal{P}_{[u, w]}(h, \bar{h})=\frac{|w-u|}{|w-u|} \int_{0}^{1}\left|(1-t) u+t w-\frac{u+w}{2}\right|^{2} d t \\
\quad-\left|\frac{|w-u|}{|w-u|} \int_{0}^{1}\left((1-t) u+t w-\frac{u+w}{2}\right) d t\right|^{2} \\
=|w-u|^{2} \int_{0}^{1}\left(t-\frac{1}{2}\right)^{2} d t-|w-u|^{2}\left|\int_{0}^{1}\left(t-\frac{1}{2}\right) d t\right|^{2}=\frac{1}{12}|w-u|^{2} .
\end{gathered}
$$

Based on the calculations outlined above, we can state the following result as well:

Proposition 11 Assume that $f$ is holomorphic in $G$, an open domain and suppose $[u, w] \subset G$. If $f^{\prime} \in \bar{\Delta}_{[u, w]}(\theta, \Theta)$ for some $\theta, \Theta \in \mathbb{C}$ with $\theta \neq \Theta$, then

$$
\begin{aligned}
& \left|\frac{f(u)+f(w)}{2}-\frac{1}{w-u} \int_{u}^{w} f(z) d z\right| \\
& \leq \frac{\sqrt{3}}{6}|w-u|\left(\frac{1}{4}|\Theta-\theta|^{2}-\left|\frac{f(w)-f(u)}{w-u}-\frac{\theta+\Theta}{2}\right|^{2}\right)^{1 / 2} \\
& \quad \leq \frac{\sqrt{3}}{12}|w-u||\Theta-\theta| .
\end{aligned}
$$

Since

$$
\begin{aligned}
\operatorname{Re}\left[\left(\Phi-\frac{f(w)-f(u)}{w-u}\right)\left(\overline{\frac{f(w)-f(u)}{w-u}}-\bar{\phi}\right)\right] \\
=\frac{1}{4}|\Phi-\phi|^{2}-\left|\frac{f(w)-f(u)}{w-u}-\frac{\phi+\Phi}{2}\right|^{2},
\end{aligned}
$$

then the inequality (37) can also be written as

$$
\begin{aligned}
& \left|\frac{f(u)+f(w)}{2}-\frac{1}{w-u} \int_{u}^{w} f(z) d z\right| \\
& \leq \frac{\sqrt{3}}{6}|w-u|\left(\operatorname{Re}\left[\left(\Phi-\frac{f(w)-f(u)}{w-u}\right)\left(\frac{\overline{f(w)-f(u)}}{w-u}-\bar{\phi}\right)\right]\right)^{1 / 2} \\
& \leq \frac{\sqrt{3}}{12}|w-u||\Theta-\theta|
\end{aligned}
$$


Acknowledgement. The author would like to thank the anonymous referee for valuable suggestions that have been implemented in the final version of the paper.

\section{References}

[1] M. W. Alomari, A companion of Grüss type inequality for Riemann-Stieltjes integral and applications, Mat. Vesnik, vol. 66, no. 2, 2014, 202-212.

[2] D. Andrica, C. Badea, Grüss' inequality for positive linear functionals, Period. Math. Hungar., vol. 19, no. 2, 1988, 155-167.

[3] D. Baleanu, S. D. Purohit, F. Uçar, On Grüss type integral inequality involving the Saigo's fractional integral operators, J. Comput. Anal. Appl., vol. 19, no. 3, 2015, 480-489.

[4] P. L. Chebyshev, Sur les expressions approximatives des intègrals dèfinis par les outres prises entre les même limites, Proc. Math. Soc. Charkov, vol. 2, 1882, 93-98.

[5] P. Cerone, On a Čebyšev-type functional and Grüss-like bounds, Math. Inequal. Appl., vol. 9, no. 1, 2006, 87-102.

[6] P. Cerone, S. S. Dragomir, A refinement of the Grüss inequality and applications, Tamkang J. Math., vol. 38, no. 1, 2007, 37-49. Preprint RGMIA Res. Rep. Coll., vol. 5, no. 2, 2002, Article 14.

[7] P. Cerone, S. S. Dragomir, Some new Ostrowski-type bounds for the Čebyšev functional and applications, J. Math. Inequal., vol. 8, no. 1, 2014, 159-170.

[8] P. Cerone, S. S. Dragomir, J. Roumeliotis, Grüss inequality in terms of $\Delta$ seminorms and applications, Integral Transforms Spec. Funct., vol. 14, no. 3, 2003, 205-216.

[9] X. L. Cheng, J. Sun, A note on the perturbed trapezoid inequality, J. Ineq. Pure and Appl. Math., vol. 3, no. 2, art. 29, 2002.

[10] S. S. Dragomir, A generalization of Grüss's inequality in inner product spaces and applications, J. Math. Anal. Appl., vol. 237, no. 1, 1999, 74-82.

[11] S. S. Dragomir, A Grüss' type integral inequality for mappings of r-Hölder's type and applications for trapezoid formula, Tamkang J. Math., vol. 31, no. 1, 2000, 43-47.

[12] S. S. Dragomir, Some integral inequalities of Grüss type, Indian J. Pure Appl. Math., vol. 31, no. 4, 2000, 397-415.

[13] S. S. Dragomir, Integral Grüss inequality for mappings with values in Hilbert spaces and applications, J. Korean Math. Soc., vol. 38, no. 6, 2001, 1261-1273. 
[14] S. S. Dragomir, I. A. Fedotov, An inequality of Grüss' type for Riemann-Stieltjes integral and applications for special means, Tamkang J. Math., vol. 29, no. 4, 1998, 287-292.

[15] S. S. Dragomir, I. Gomm, Some integral and discrete versions of the Grüss inequality for real and complex functions and sequences, Tamsui Oxf. J. Math. Sci., vol. 19, no. 1, 2003, 67-77.

[16] A. M. Fink, A treatise on Grüss' inequality, Analytic and Geometric Inequalities and Applications, 93-113, Math. Appl., 478, Kluwer Acad. Publ., Dordrecht, 1999.

[17] G. Grüss, Über das Maximum des absoluten Betrages von $\frac{1}{b-a} \int_{a}^{b} f(x) g(x) d x-$ $\frac{1}{(b-a)^{2}} \int_{a}^{b} f(x) d x \int_{a}^{b} g(x) d x$, Math. Z., vol. 39, 1935, 215-226.

[18] D. Jankov Maširević, T. K. Pogány, Bounds on Čebyšev functional for $C_{\varphi}[0,1]$ function class, J. Anal., vol. 22, 2014, 107-117.

[19] Z. Liu, Refinement of an inequality of Grüss type for Riemann-Stieltjes integral, Soochow J. Math., vol. 30, no. 4, 2004, 483-489.

[20] Z. Liu, Notes on a Grüss type inequality and its application, Vietnam J. Math., vol. 35, no. 2, 2007, 121-127.

[21] A. Lupaş, The best constant in an integral inequality, Mathematica (Cluj, Romania), 15, vol. 38 , no. 2, 1973, 219-222.

[22] A. Mc. D. Mercer, P. R. Mercer, New proofs of the Grüss inequality, Aust. J. Math. Anal. Appl., vol. 1, no. 2, 2004, art. 12.

[23] N. Minculete, L. Ciurdariu, A generalized form of Grüss type inequality and other integral inequalities, J. Inequal. Appl., 2014, 2014:119, 18 pp.

[24] A. M. Ostrowski, On an integral inequality, Aequat. Math., vol. 4, 1970, 358373.

[25] B. G. Pachpatte, A note on some inequalities analogous to Grüss inequality, Octogon Math. Mag., vol. 5, no. 2, 1997, 62-66.

[26] J. Pečarić, Š. Ungar, On a inequality of Grüss type, Math. Commun., vol. 11, no. $2,2006,137-141$.

[27] M. Z. Sarikaya, H. Budak, An inequality of Grüss like via variant of Pompeiu's mean value theorem, Konuralp J. Math., vol. 3, no. 1, 2015, 29-35.

[28] N. Ujević, A generalization of the pre-Grüss inequality and applications to some quadrature formulae, J. Inequal. Pure Appl. Math., vol. 3, no. 1, 2002, art. 13, 9 pp. 


\section{Silvestru Sever Dragomir}

Victoria University

College of Engineering and Science

Melbourne City, MC 8001, Australia

e-mail: Sever.Dragomir@vu.edu.au 